\title{
Recognition of tramadol abuse, dispensing practices, and opinions about its control policy among community pharmacists in Bangkok, Thailand
}

Tulaya Potaros ${ }^{1}$, Suwimon Yeephu ${ }^{2, *}$

Abstract

Background: Tramadol is classified as a pharmacist-only (restricted) medicine by the Food and Drug Administration of Thailand (Thai FDA). Because of concern about its abuse, in September 2013 the Thai FDA announced a policy to control the distribution of tramadol in community pharmacies.

Objectives: To identify tramadol dispensing practices by community pharmacists in Bangkok, their recognition of tramadol abuse and the Thai FDA control policy announcement; and opinions about the tramadol control policy.

Methods: This descriptive cross-sectional study was conducted in community pharmacies located in Bangkok. Pharmacists on duty were interviewed from September 2015 to April 2016.

Results: Data from 305 pharmacists working in 305 pharmacies revealed that tramadol, both single (tramadol alone) and combination (tramadol plus acetaminophen) formulations, was available in 185 pharmacies (60.7\%). Most pharmacists dispensed tramadol to supply regular medicine along with previous prescriptions (74\%). Among 305 pharmacists, 304 (99.7\%) recognized tramadol abuse in combination with cold-cough remedies and carbonated beverages can create euphoria. Most (97.7\%) knew about the announcement of the tramadol control policy, and most (82.6\%) thought that the policy was practical. Approximately $43 \%$ of pharmacists agreed that the policy was effective in reducing the tramadol abuse problem, whereas $36.7 \%$ disagreed. Moreover, $60.3 \%$ disagreed with rescheduling tramadol as a prescription-only medicine. In their opinion, tramadol should still be available in pharmacies, to be dispensed by community pharmacists to patients with medical necessity.

Conclusions: Further studies nationwide in Thailand are likely to be useful to represent and compare information in different parts of the country.

Keywords: drug and narcotic control, Food and Drug Administration, pharmacists, tramadol, Thailand

Tramadol, a nontraditional centrally acting analgesic, possesses multimodal mechanisms of action, including as a weak $\mu$-opioid receptor agonist and a norepinephrine and serotonin reuptake inhibitor [1]. Tramadol is used to alleviate moderate-to-severe pain in various acute and chronic pain conditions $[1,2]$.

According to the Food and Drug Administration of Thailand (Thai FDA), tramadol is classified as a pharmacist-only

*Correspondence to: Suwimon Yeephu, Department of Clinical Pharmacy, Faculty of Pharmacy, Srinakharinwirot University, Nakhon Nayok 26120, Thailand, e-mail: suwimony@g.swu.ac.th

'Department of Biopharmacy, Faculty of Pharmacy, Srinakharinwirot University, Nakhon Nayok 26120, Thailand 2Department of Clinical Pharmacy, Faculty of Pharmacy, Srinakharinwirot University, Nakhon Nayok 26120, Thailand

¿ Open Access. ๑ 2018 Tulaya Potaros, Suwimon Yeephu published by Sciendo. (cc) Br-Nc-ND This work is licensed under the Creative Commons Attribution NonCommercial-NoDerivatives 4.0 License. 
medicine or "dangerous drug" listed under Thai Medicine Act BE 2510 (1967), which can be dispensed by licensed pharmacists in community pharmacies [3]. A total daily dose of no more than $400 \mathrm{mg}$ is usually recommended [2]. Concerning its safety, especially at higher doses, tramadol has the potential to produce euphoric effects and serotonin syndrome [4]. One long-term study in patients with chronic noncancer pain demonstrated that the abuse prevalence of tramadol and nonsteroidal anti-inflammatory drugs (NSAIDs) was equivalent, with both significantly less than the rate for hydrocodone [5]. This result indicated that the therapeutic use of tramadol rarely leads to drug abuse in patients without an active substance abuse problem [5]. Although the potential for tramadol abuse is low, various countries report data relating to its abuse among their populations [3]. Misuse or abuse of tramadol and long-term use of high-dose or concomitant use with alcoholic beverages can cause side effects in the central nervous system such as euphoria, convulsion, and unconsciousness [6]. In Thailand, the Princess Mother National Institute on Drug Abuse Treatment (PMNIDAT), in the Department of Medical Services, Ministry of Public Health, reported an increasing incidence of patients with tramadol addiction from 5 cases in 2014 to 46 cases in 2016. These data were in accord with the rate of $4 \times 100$ cocktail-addicted patients, which rose from 50 to 114 cases, respectively [7]. The $4 \times 100$ cocktail is a combination of 4 ingredients including boiled kratom leaves, a cola-type soft drink, codeine- or diphenhydramine-containing cough syrup, and agents with narcotic effects such as tramadol, anxiolytic drugs, mosquito coils, and herbicides [8]. In July 2015, an incident of tramadol misapplication in a Thai schoolchild was reported. After taking a combination of an overdose of tramadol and promethazine syrup, the student developed severe convulsions [9]. Currently, abuse and misuse of over-the-counter drugs have increased dramatically, leading to worldwide public health problems. One study in Yemen indicated that tramadol is among the top 4 suspected abuse medications in community pharmacies [10]. In Thailand, tramadol can be dispensed in community pharmacies, which are probably one source of seeking the drug for misuse or abuse.

Because of its abuse and potential for dependence, especially among young people and adolescents, the Thai FDA has announced a restrictive control policy on tramadol dispensing in community pharmacies since September 2013. This policy comprises 6 important rules for dispensing tramadol as follows. First, tramadol can be dispensed only by pharmacist on duty. Second, tramadol, in any case, dispensing to children aged $<17$ years is prohibited. Third, tramadol can be dispensed only to patients in case of medical necessity with an amount of not $>20$ tablets/capsules per patient. Fourth, the licensee and pharmacist on duty have to make a record of the tramadol purchased or sold truly, correctly, and currently. Fifth, the pharmacist on duty is required to sign a purchase document in case of a tramadol purchase order from the manufacturer and importer. Finally, the monthly order quantity is limited to not more than 1,000 tablets/capsules per pharmacy [11].

Bangkok, the capital city of Thailand, is a high-risk area for widespread drug abuse or substance-related disorders [12]. In this region, the perspective of community pharmacists on the current state of tramadol use and the Thai FDA control policy is not well documented. These data are needed for a better understanding of the tramadol situation in Bangkok, which could be beneficial for pharmacists nationwide, other health care professionals, and policy makers. The objectives of this study were to identify the tramadol dispensing practices by community pharmacists in Bangkok, their recognition of current situation related to tramadol abuse, and the control policy announcement; and their opinions of the tramadol control policy of the Thai FDA.

\section{Materials and methods}

The protocol for this descriptive cross-sectional study was approved by the ethics committee of the Faculty of Pharmacy at Srinakharinwirot University (approval No. 007/2558). The data collection was conducted by interviewing licensed pharmacists working in community pharmacies situated in Bangkok, Thailand, from September 2015 to April 2016.

Based on the 4,794 pharmacies located in Bangkok [13], the sample size calculated using the Taro Yamane formula [14] with a $95 \%$ confidence interval was 370 . A proportional quota sampling strategy focusing on utility areas in Bangkok was utilized [15]. In the present study, the capital was divided into 6 utility areas including residential, agricultural, industrial, commercial, art and cultural, and official places, which covered most of the land use in Bangkok. Each area comprised various administrative districts, which were selected by convenience sampling. In each administrative district, convenience sampling was then performed for the sample selection. The pharmacists on duty were approached by direct contact without making appointments in advance. Community pharmacists with work experience of $<3$ months were excluded. Then, the nature of the study including the rationale, objectives, and the interview process and time was explained to each potential participant. By implied consent, community pharmacists willing to participate in this study were interviewed to collect the information.

A structured interview form containing a checklist and short answer questions was developed to collect the data in this study. To develop and validate the interview form, content 
validation by 4 experts (1, provincial public health pharmacist; 2, legal expert pharmacist, assistant professor from the Faculty of Pharmacy at Srinakharinwirot University; 3, legal expert pharmacist with work experience at provincial public health office, assistant professor from the Faculty of Pharmacy at Srinakharinwirot University; 4, community pharmacist with work experience involving primary pharmaceutical services $>5$ years) and cognitive interview by 5 community pharmacists working in the Nakhon Nayok province were performed. The data collection consisted of 4 parts as follows.

- Part 1. The demographic data and the characteristics of pharmacists (sex, age, position as full-time or part-time pharmacists, and duration of work experience in pharmacies) and pharmacies (type of business, type of business channel, number of daily customers, number of daily customers who had pain or asked for analgesic drugs, and the availability of tramadol).

- $\quad$ Part 2. Dispensing practices of tramadol during the previous 3 months as measured by 5 questions as follows: (1) quantity of tramadol dispensed (number of patients per week and number of tablets/capsules per patient); (2) the major reasons and indications for dispensing; (3) recommendations about the dosage regimen; (4) recommendations regarding side effects; and (5) recommendations about special precautions.

- Part 3. Recognition by pharmacists of the current situation of tramadol abuse and the announcement on tramadol control policy, as measured by 6 questions as follows: (1) know or do not know about tramadol abuse potential; (2) sources of information about tramadol abuse; (3) know or do not know about the announcement by the Thai FDA on control policy; (4) sources of information about the control policy; (5) the experience of pharmacists with teenagers asking for tramadol at their pharmacies; and (6) the experience of pharmacists with customers asking for $>20$ tramadol tablets/capsules per visit.

- Part 4. The opinions of the pharmacists on tramadol control policy were assessed by 3 questions as follows: (1) the practicability of policy implementation; (2) the effectiveness of the policy to reduce any tramadol abuse problem; and (3) the scenario of rescheduling tramadol as a prescription medicine.

With regard to the consistency of interview process, an interview guide was developed. The interviewers were informed about the procedure, and the interview process was rehearsed.

Descriptive statistics were used to describe the characteristics and features of the data. The basic descriptions of the quantitative variables are presented as percentage and mean \pm standard deviation (SD). The questions for which there was no response were considered as missing values and omitted from the analysis.

\section{Results}

Researchers contacted 906 pharmacies in Bangkok. During the visits, 357 pharmacies (39.4\%) had pharmacists on duty. Of the 357 pharmacists, 52 refused to participate in the study, because they had insufficient time to answer the questions. Therefore, 305 pharmacists $(85.4 \%)$ were interviewed to collect the information.

\section{Demographic data and characteristics of pharmacists and pharmacies}

The demographic data and characteristics of pharmacists and pharmacies are summarized in Table 1. Most pharmacists were women $(62.3 \%)$ with a mean \pm SD age of $36.6 \pm 11.5$ years (range 24-73 years). About $70 \%$ of pharmacies were standalone businesses. Almost half of the pharmacies (40.7\%) had customers who came with pain symptoms or asked for analgesic drugs at a rate of 11-20 cases daily. Of these 305 pharmacies, tramadol was available in $185(60.7 \%)$ in 2 formulations, as a single drug $(50 \mathrm{mg})$ and as a fixed combination (tramadol $37.5 \mathrm{mg}$ plus acetaminophen [paracetamol] $325 \mathrm{mg}$ ). The availability of tramadol at the 185 pharmacies where it was available was $16 \%$ as a single drug only, $52 \%$ as a combination drug only, and $31 \%$ as both formulations.

Considering 120 pharmacies with unavailable tramadol, more than half of the pharmacists $(57 \%)$ working at these pharmacies claimed that tramadol was withdrawn from their drug list after the announcement of the Thai FDA control policy. Another key reason for unavailable tramadol was to avoid drug abuse especially by teenagers (39\%).

\section{Dispensing practices of tramadol}

With regard to pharmacists working in pharmacies with tramadol availability, a little less than half (48\%) of pharmacists dispensed the single formulation to $1-5$ customers per week and $54 \%$ of pharmacists dispensed the combined drug to $<1$ customer per week. The highest dispensed amount of either formulation was 10-20 tablets/capsules per patient as summarized in Table 2.

Community pharmacists dispensed tramadol when supplying regular medicine along with previous prescriptions, for moderate-to-severe pain, and for the ineffectiveness of other 
Table 1. Demographic data and characteristics of pharmacists and pharmacies

\begin{tabular}{|c|c|}
\hline Characteristics & Values $^{\dagger}$ \\
\hline \multicolumn{2}{|l|}{ Pharmacists $(n=305)$} \\
\hline Female & $190(62.3)$ \\
\hline Age: mean $\pm S D$ (years) & $36.6 \pm 11.5$ \\
\hline Full-time pharmacist & $238(78.0)$ \\
\hline Work experience in pharmacies: mean \pm SD (years) & $8.8 \pm 9.3$ \\
\hline \multicolumn{2}{|l|}{ Pharmacies $(n=305)$} \\
\hline \multicolumn{2}{|l|}{ Type of business } \\
\hline Stand-alone pharmacies & $216(70.8)$ \\
\hline Chain pharmacies & $89(29.2)$ \\
\hline \multicolumn{2}{|l|}{ Type of business channel } \\
\hline Retail pharmacies & $279(91.5)$ \\
\hline Both retail and wholesale pharmacies & $26(8.5)$ \\
\hline \multicolumn{2}{|l|}{ Number of customers (cases per day) } \\
\hline$\leq 20$ & $20(6.6)$ \\
\hline $21-40$ & $69(22.6)$ \\
\hline $41-60$ & $56(18.4)$ \\
\hline $61-80$ & $47(15.4)$ \\
\hline $81-100$ & $61(20.0)$ \\
\hline$>100$ & $52(17.1)$ \\
\hline \multicolumn{2}{|l|}{$\begin{array}{l}\text { Number of customers who had pain or asked for analgesics } \\
\text { (cases per day) }\end{array}$} \\
\hline$\leq 10$ & $82(26.9)$ \\
\hline $11-20$ & $124(40.7)$ \\
\hline $21-30$ & $48(15.7)$ \\
\hline $31-40$ & $25(8.2)$ \\
\hline $41-50$ & $20(6.6)$ \\
\hline$>50$ & $6(2.0)$ \\
\hline \multicolumn{2}{|l|}{ Availability of tramadol } \\
\hline Yes & $185(60.7)$ \\
\hline No & $120(39.3)$ \\
\hline \multicolumn{2}{|l|}{ Formulation of tramadol available in 185 pharmacies } \\
\hline Single drug (tramadol) only & $30(16)$ \\
\hline Fixed combination drug (tramadol/acetaminophen) only & $97(52)$ \\
\hline Both formulations & $58(31)$ \\
\hline
\end{tabular}

${ }^{\dagger}$ Values are $\mathrm{n}(\%)$ unless otherwise indicated

$\mathrm{SD}$, standard deviation

analgesic drugs; the proportions of major indications for dispensing tramadol are summarized in Table $\mathbf{2}$.

Data regarding recommendations about the dosage regimen of single drug and combined drug was given by 88 and 155 pharmacists, respectively. Pharmacists often recommended the patients take a dosage regimen of 1 tablet or capsule every $6-8$ hours as needed or as directed by their physicians ( $28 \%$ for each formulation), 1 tablet or capsule 3 times daily after meals (26\% for the single drug and $19 \%$ for the combined formulation), 1 tablet or capsule 2 times daily after meals ( $14 \%$ for the single drug and $11 \%$ for the combined
Table 2. Tramadol dispensing practices

\begin{tabular}{cc}
\hline Tramadol dispensing & $\begin{array}{c}\text { Number of pharmacies } \\
\text { pharmacists } \mathbf{n}(\%)\end{array}$ \\
\hline
\end{tabular}

Dispensing of single drug (tramadol)

Number of customers (cases per week;

$\mathrm{n}=83)^{\dagger}$

$<1$

$1-5$

$25(30)$

40 (48)

$11(13)$

$6-10$

$>10$

7 (8)

Number of drugs dispensed

(tablets/capsules per patient; $\mathrm{n}=75$ ) $^{\ddagger}$

$<10$

$4(5)$

$10-20$

$69(92)$

$>20$

2 (3)

Dispensing of fixed combination drug (tramadol plus acetaminophen)

Number of customers (cases per week; $\mathrm{n}=136)^{5}$

$\begin{array}{lr}<1 & 74(54) \\ 1-5 & 55(41) \\ 6-10 & 4(3) \\ >10 & 3(2)\end{array}$

Number of drugs dispensed

(tablets/capsules per patient; $\mathrm{n}=113$ )"

$<10$

7 (6)

$10-20$

$105(93)$

$>20$

1 (1)

Major reasons for dispensing $(\mathbf{n}=\mathbf{1 8 5})$

Supplying regular medicine along with

previous prescriptions $137(74)$

Moderate-to-severe pain $112(61)$

Ineffectiveness of other analgesic drugs 94 (51)

Allergy to other analgesic drugs $\quad 56$ (30)

Precaution/contraindication to other

analgesics, e.g., renal disease and peptic

ulcer

$30(16)$

Combining with other analgesics, e.g.,

NSAIDs, acetaminophen, and muscle

relaxants

$28(15)$

Major indications for dispensing $(n=185)$

Bone pain

$107(58)$

Cancer pain

$78(42)$

Back pain

$72(39)$

$56(30)$

$\begin{array}{ll}\text { Tooth pain/pain in oral cavity } & 31(17) \\ \text { Neuropathic pain } & 31(17)\end{array}$

Number of pharmacists giving an

explanation to customers $(n=185)$

Explanation of potential side effect(s)

$155(84)$

Explanation of special precaution(s)

$96(52)$

Major side effect(s) explained $(n=155)$

Nausea/vomiting

Drowsiness/somnolence $77(50)$

Dizziness $71(46)$

Constipation $50(32)$ 


\begin{tabular}{lc}
\hline Tramadol dispensing & $\begin{array}{c}\text { Number of pharmacies/ } \\
\text { pharmacists } \mathbf{n}(\%)\end{array}$ \\
\hline $\begin{array}{l}\text { Major special precaution(s) explained } \\
(\mathbf{n}=\mathbf{9 6})\end{array}$ & \\
Take only as required for pain & $32(33)$ \\
Be aware of drug addiction & $25(26)$ \\
Avoid long-term use & $18(19)$ \\
Be aware of drug interaction & $9(9)$ \\
\hline
\end{tabular}

tThe number of pharmacies with tramadol availability was 88 , and the missing data were 5 , then $n=83$

${ }^{*}$ The number of pharmacies with tramadol availability was 88 , and the missing data were 13 , then $n=75$

${ }^{5}$ The number of pharmacies with fixed combination drug availability was 155 , the missing data were 19 , then $n=136$

"The number of pharmacies with fixed combination drug was 155 , the missing data were 42 , then $n=113$

NSAIDs, nonsteroidal anti-inflammatory drugs

formulation), or 1 tablet or capsule 3 times daily after meals when required for pain (13\% for the single drug and $12 \%$ for the combined formulation).

When dispensing tramadol to the customers, most (155; 84\%) pharmacists explained its potential side effects, including nausea/vomiting and drowsiness or somnolence and approximately half $(96 ; 52 \%)$ of pharmacists informed patients about some special precautions, such as taking tramadol only as required for pain and to be aware of the potential for drug addiction and avoid long-term use as summarized in Table 2.

\section{Recognition of tramadol abuse and announcement of the tramadol control policy}

Among community pharmacists surveyed, 304 (99.7\%) knew about tramadol abuse from various sources, such as television news, social media, and the Thai FDA as summarized in Table 3. The pharmacists claimed that tramadol was used, particularly by teenagers, in combination with cough and cold remedies (such as antihistamines or cough syrup), carbonated (cola) beverages, and/or kratom (also known as $4 \times 100$ drug cocktail) to produce euphoric effects.

Among these pharmacists, 298 (97.7\%) acknowledged the announcement of the Thai FDA control policy on tramadol dispensing in pharmacies. Most pharmacists received the information from a letter from the Thai FDA. The other main sources of information were social media and the head office of pharmacy chain stores as summarized in Table 3.

In the experience of the pharmacists surveyed, 215 pharmacists $(70.5 \%)$ claimed that teenagers sought tramadol from pharmacies, with the most frequent experience being of 1-5 cases per week as summarized in Table 3. Young people
Table 3. Recognition of current tramadol abuse and announcement of the tramadol control policy

\begin{tabular}{|c|c|}
\hline Recognition by pharmacists & n (\%) \\
\hline \multicolumn{2}{|l|}{ Tramadol abuse situation } \\
\hline \multicolumn{2}{|l|}{ Recognitions $(n=305)$} \\
\hline Know & $304(99.7)$ \\
\hline Do not know & $1(0.3)$ \\
\hline \multicolumn{2}{|l|}{ Major sources of information ( $n=304)$} \\
\hline Television news & $150(49.3)$ \\
\hline Social media & $135(44.4)$ \\
\hline Thai FDA & $112(36.8)$ \\
\hline Newspapers & $108(35.5)$ \\
\hline Patients/customers & $56(18.4)$ \\
\hline Other pharmacists & $45(14.8)$ \\
\hline \multicolumn{2}{|l|}{ Announcement of tramadol control policy } \\
\hline \multicolumn{2}{|l|}{ Recognitions $(n=305)$} \\
\hline Know & $298(97.7)$ \\
\hline Do not know & $7(2.3)$ \\
\hline \multicolumn{2}{|l|}{ Major sources of information ( $n=298$ ) } \\
\hline Thai FDA & $207(69.5)$ \\
\hline Social media & $52(17.5)$ \\
\hline Head offices of pharmacy chain stores & $33(11.1)$ \\
\hline Other pharmacists & $25(8.4)$ \\
\hline Television news & $12(4.0)$ \\
\hline Faculties of pharmacy & $12(4.0)$ \\
\hline \multicolumn{2}{|c|}{$\begin{array}{l}\text { The experiences of pharmacists with teenagers asking } \\
\text { for tramadol }(n=305)\end{array}$} \\
\hline Yes & $215(70.5)$ \\
\hline \multicolumn{2}{|c|}{ Number of teenagers (cases per week; $n=213)^{+}$} \\
\hline$<1$ & $72(33.8)$ \\
\hline $1-5$ & $81(38.0)$ \\
\hline $6-10$ & 29 (13.6) \\
\hline$>10$ & $31(14.6)$ \\
\hline \multicolumn{2}{|c|}{$\begin{array}{l}\text { The experiences of pharmacists with customers asking } \\
\text { for tramadol }>20 \text { tablets/capsules per case }(n=305)\end{array}$} \\
\hline Yes & $102(33.4)$ \\
\hline \multicolumn{2}{|c|}{ Number of customers (cases per week; $n=94)^{\ddagger}$} \\
\hline$<1$ & $70(75)$ \\
\hline $1-5$ & $19(20)$ \\
\hline $6-10$ & $2(2)$ \\
\hline$>10$ & $3(3)$ \\
\hline
\end{tabular}

'The number of pharmacists with experience of teenagers asking for tramadol was 215, and the missing data were 2 , then $n=213$

*The number of pharmacists with experience of customers asking for tramadol more than 20 tablets/capsules per case was 102, and the missing data were 8 , then $n=94$

Thai FDA, Food and Drug Administration of Thailand

requested tramadol using the following phrases: "greenyellow medicine" (29.8\%); "tramadol" (26.3\%); "greenyellow capsule" (12.3\%); and "green-yellow analgesic" $(8.2 \%)$. However, most pharmacists $(98.6 \%)$ claimed that they refused to dispense tramadol to young customers with the main reason being that they considered tramadol would be misused. 
In addition, $102(33.4 \%)$ of the community pharmacists surveyed experienced customers requesting more than 20 tablets/ capsules per visit, with the most frequent request of $<1$ case per week as summarized in Table 3. Of these 102 pharmacists, most denied dispensing because of the control policy of the Thai FDA. By contrast, 22 pharmacists (21.6\%) provided more than 20 tablets/capsules as requested when supplying regular medicine along with previous prescriptions for the customers.

\section{Opinions of the Thai FDA control policy on dispensing tramadol}

In the opinion of the 305 community pharmacists surveyed, $252(82.6 \%)$ considered that, in general, following the Thai FDA policy was practical (Table 4). In compliance with the tramadol control policy, some of the following rules were considered difficult to implement: prohibition on dispensing to children aged $<17$ years ( $15.7 \%$ pharmacists); limitation of amount dispensed to not $>20$ tablets/capsules per customer (17.7\% pharmacists); and recording the amount of tramadol purchased or sold ( $22.6 \%$ pharmacists).

The control policy to decrease the tramadol abuse problem was considered effective by $131(43.0 \%)$ of the 305 pharmacists surveyed, while $112(36.7 \%)$ pharmacists disagreed (Table 4). The main reason for their agreement was that this policy can limit improper drug access. Those who disagreed claimed that this policy focused on dispensing practices by community pharmacists while it could not control the accessibility of tramadol from other sources, such as social media, grocery, or pharmacies with no pharmacists on duty. In addition, the policy still had weak points, for example, the customers could repeatedly purchase tramadol from many pharmacies in a single day, the abusers may be older than 17 years

Table 4. Opinions of pharmacists on tramadol control policy

\begin{tabular}{lr}
\hline Opinion of pharmacists & $\mathbf{n}(\%)$ \\
\hline Practicability of policy implementation $(\mathrm{n}=305)$ & \\
$\quad$ Practical & $252(82.6)$ \\
$\quad$ Not practical & $53(17.4)$ \\
Ability of policy to reduce tramadol abuse problem & \\
$(\mathrm{n}=305)$ & \\
$\quad$ Agreed & $131(43.0)$ \\
$\quad$ Disagreed & $112(36.7)$ \\
$\quad$ Not assured & $62(20.3)$ \\
Scenario of rescheduling tramadol as a prescription & \\
medicine ( $=305)$ & \\
$\quad$ Agreed & \\
Disagreed & $184(29.5)$ \\
$\quad$ Not assured & $31(10.2)$ \\
\hline
\end{tabular}

or teenagers with substance-related disorders or who intended to abuse tramadol could ask other people to buy tramadol in pharmacies. Many pharmacists proposed that providing education, especially to teenagers, might be a better way to reduce tramadol abuse problems.

A majority of pharmacists $(184 ; 60.3 \%)$ disagreed that tramadol would be less available for abuse if it was rescheduled as a prescription-only medicine (Table 4). The key reason for disagreement with rescheduling was that this drug is still necessary for some patients in their community, including patients with moderate-to-severe pain, ineffectiveness or contraindications to other analgesics, and limitations of time and budget to go to a hospital to get a prescription. Therefore, pharmacists should have the authority to dispense tramadol to these patients in their pharmacies. By contrast, 90 (29.5\%) pharmacists agreed with rescheduling. They explained that if pain was too severe to treat with simple analgesics, tramadol should be considered by physicians in hospitals. Moreover, they considered that if dispensing tramadol without prescription is prohibited in community pharmacies, the tramadol abuse problem could be solved.

\section{Discussion}

The present study provided 3 main findings relating to tramadol, which included dispensing practices, the recognition of tramadol abuse and the announcement of the Thai FDA control policy, and opinions about the control policy among community pharmacists in Bangkok, Thailand.

Of 185 pharmacies with tramadol availability, the formulation of fixed-dose combination (tramadol $37.5 \mathrm{mg}$ plus acetaminophen $325 \mathrm{mg}$ ) was found to be the preferred analgesic compared with the single formulation. This might be due to the good efficacy and tolerability profile of the combined drugs. Tramadol plus acetaminophen can provide a synergistic analgesic effect [16]. One study in postoperative pain management demonstrated the comparable analgesic efficacy and fewer adverse events with a fixed-dose combination compared with tramadol $50 \mathrm{mg}$ monotherapy. Therefore, the combined drug could reduce tramadol consumption by $24 \%$ [17]. Moreover, as superior to ad hoc dose ratios, the fixed-ratio combination of tramadol plus acetaminophen provided standardized reproducible improvement in pain scores and side effect profiles in multicenter randomized controlled trials for the management of some chronic pain conditions [18]. Moreover, community pharmacists participating in this study revealed that most young people requested tramadol by using phrases such as "green-yellow medicine", "tramadol", "green-yellow capsule", and "green-yellow analgesics", which reflects the 
preferred abuse formulation as a single rather than a combined drug. This indicated the high abuse potential of the single drug formulation among Thai teenagers, which is one reason for the withdrawal of tramadol from the list of available drugs in many pharmacies involved in the present study.

We found that tramadol was dispensed to some customers as a single drug and combined with acetaminophen to others, indicating that the 2 formulations should be available in pharmacies. The dispensed amounts were mostly 10-20 tablets/capsules per customer, which was consistent with the rule of limitations on sale amounts (not more than 20 tablets/ capsules per case) as established by the tramadol control policy. Tramadol was dispensed to alleviate moderate-to-severe pain as recommended by the Thai FDA [3]. Pharmacists suggested various dosage regimens of tramadol to customers depending on their assessment of individual customers, but did not exceed the maximum recommended dosage. Most pharmacists explained the common side effects, indicating good dispensing practice related to this issue. However, only a small number of pharmacists provided an explanation of special precautions related to drug interactions. Tramadol has the potential to interact with numerous drugs such as tricyclic antidepressants, selective serotonin reuptake inhibitors, serotonin norepinephrine reuptake inhibitors, and drugs affecting cytochrome P450 2D6 (CYP2D6) activity [19, 20]. We recommend pharmacists remind themselves of the possible harmful effects of drug interactions and inform patients about the interactions before dispensing tramadol.

Pharmacists received information about tramadol being a drug of abuse among Thai youth from various sources, but especially from television news and social media. This information reminds pharmacists to realize the current potential for abuse and encourage them to prevent problems. However, the widespread distribution of this information probably leads to imitation of the behavior by teenagers. The authorities could directly publicize more information about the dangers of tramadol abuse to the community through effective communicative channels, including television, social media, and newspapers.

Most pharmacists knew about the announcement of tramadol control policy, mostly through the letter from the Thai FDA, indicating effective public relations. However, to remind community pharmacists, the announcement period should be longer and continuously supported through other appropriated sources, such as social media, professional associations, and educational institutes.

Most of the community pharmacists $(70.5 \%)$ had encountered children and teenagers asking for tramadol at their pharmacies and suspected intended tramadol abuse. This finding is consistent with the findings of Zabihi et al. that $>55 \%$ of the customers seeking tramadol were aged $<18$ years [21]. The pharmacists in this study refused to supply tramadol to young customers because of the high risk of intended drug abuse. Some pharmacists also noticed that if tramadol was dispensed to one teenager, others would also request tramadol from their particular community pharmacy. This appears consistent with a previous report that the motivations of peers played a pivotal role in substance abuse among adolescents [22].

The results from the interviews indicated that most community pharmacists dispensed tramadol in accordance with Thai FDA control policy compliant with the aspects of age and quantity limitations. However, screening the tendency for tramadol abuse by customers using other questions, as mentioned in the report by Zabihi et al. [21] including "What are you using tramadol for?", "Have you ever needed to increase your tramadol dose without checking with your physicians?" and "Have you ever felt that you could not stop or decrease your use of tramadol?", could be useful to reduce or avoid tramadol abuse in the community.

Most of the community pharmacists surveyed in this study considered that, in general, following the Thai FDA control policy was practical. Santisetthsin and Thananithisak used questionnaires sent by mail to pharmacists in Bangkok and also found that community pharmacists agreed with the tramadol control policy [23]. Although the methodology for data collection in this study and that by Santisetthsin and Thananithisak was different, the findings from both studies suggested that most pharmacists working in Bangkok accepted the implementation of the Thai FDA policy to control the sale of tramadol in community pharmacies. We found that the pharmacists participating in the present study had various opinions about whether or not the Thai FDA rule could reduce tramadol abuse. This, at least partly, reflected their lack of confidence with the effectiveness of the policy. To strengthen its implementation, an annual assessment of policy achievement to reduce tramadol abuse is proposed, such as assessment of any reduction in the number of people hospitalized because of tramadol intoxication.

The scheduling of tramadol as a controlled substance is a method to diminish abuse problems [23]. A study conducted in Kentucky and Arkansas established a reduction in the number of cases of tramadol consumption and exposure to toxicity following its addition to the schedule of controlled substances [24]. However, community pharmacists in Thailand from both the present study and that by Santisetthsin and Thananithisak mostly disagreed with rescheduling tramadol as a prescription-only medicine [23]. They opined that the lack of availability of tramadol in pharmacies would diminish the potential drug accessibility by some patients with medical necessity. Currently, tramadol remains available in community pharmacies as the Narcotics Control Board 
(NCB) Secretary General noted that a sales ban would likely affect the general public [12].

School-based education is considered an important tool to prevent drug abuse [25]. Based on the opinions of many pharmacists participating in the present study, social regulations are effective strategies to decrease the tramadol abuse. Raising awareness at school and in the community on the prevention of initial tramadol use by providing education to children and their parents is critical. Any community pharmacists acting as retailers of drugs of abuse must be strictly and legally prosecuted. Finally, cooperation between adolescents, their families, teachers, health care providers, and the government is necessary to solve this critical problem.

The present study offers important and unique data related to the perspective of community pharmacists on tramadol usage and the current control policy related to the dispensing of tramadol in the Thai capital. We consider that it contributes to a better understanding of the use and abuse of tramadol in this region, which may be of benefit to pharmacists and other health care professionals nationwide. In addition, policy makers may consider the research evidence to improve control policy to minimize the misuse or abuse of tramadol in Thailand.

The sampling technique used is a limitation of the present study. A proportional quota sampling strategy focused on the utility areas of Bangkok was used first for probability sampling, followed by a convenience sampling strategy. This method may not represent data accurately for the entire population of community pharmacists in Bangkok. The possibility of over- and under-representation of samples in some areas may affect the quality of data collection. However, we attempted to minimize this limitation by using a proportional quota sampling in the first step to cover all the utility area of Bangkok. Other potential limitations included unachieved target sample sizes, recall bias, and study locations. There were just 305 participants or $82.4 \%$ of the expected sample size. Based on the study plan, it was proposed that we could get the data from 1 pharmacist at each pharmacy. However, some pharmacists were not on duty during our visit time, which might be a result of the convenience sampling without making appointments in advance. To diminish possibility of recall bias, resulting from direct interviews with an individual pharmacist to obtain information on their past experiences, the researchers only asked for information recalled from within the previous 3 months. In the aspect of the location limitation, the study was conducted in Bangkok, which is considered to be a high-risk region for widespread drug abuse. Therefore, the findings from this city alone with its unique culture might limit the generalizability of our findings.

\section{Conclusions}

Most community pharmacists dispensed tramadol to supply regular medicine along with previous prescriptions and to customers with moderate-to-severe pain, including bone, cancer, and back pain. Almost all pharmacists knew the potential for tramadol abuse in the community, especially by teenagers, and recognized the announcement of the tramadol control policy of the Thai FDA. Almost half of them agreed that the Thai FDA control policy was effective to diminish tramadol abuse. Further studies nationwide in Thailand are likely to be useful to represent and compare information in different parts of the country.

Author contributions. Both TP and SY made substantial contributions to the conception and design of the study, the acquisition of data, and its analysis and interpretation. Both authors drafted and critically revised the manuscript, approved the final version submitted for publication, and take responsibility for statements made in the published article.

Acknowledgment. This research was supported by grant from the Faculty of Pharmacy at Srinakharinwirot University, Thailand.

Conflict of interest statement. The authors have each completed and submitted an International Committee of Medical Journal Editors Form for Disclosure Potential Conflicts of Interest. Neither of the authors has any potential conflict of interest to disclose.

\section{References}

[1] Grond S, Sablotzki A. Clinical pharmacology of tramadol. Clin Pharmacokinet. 2004; 43:879-923.

[2] Shadnia S, Soltaninejad K, Heydari K, Sasanian G, Abdollahi M. Tramadol intoxication: a review of 114 cases. Hum Exp Toxicol. 2008; 27:201-5.

[3] Narcotics control division, Ministry of public health, Thailand. Information and misuses of tramadol [online]. 2013 [cited $2016 \mathrm{Sept}$ 16]. Available from: http://narcotic.fda.moph.go.th/welcome/? $p=4326$. [in Thai]

[4] Beakley BD, Kaye AM, Kaye AD. Tramadol, pharmacology, side effects and serotonin syndrome: a review. Pain Physician. 2015; 18: 395-400.

[5] Adams EH, Breiner S, Cicero TJ, Geller A, Inciardi JA, Schnoll SH, et al. A comparison of the abuse liability of tramadol, NSAIDs, and hydrocodone in patients with chronic pain. J Pain Symptom Manage. 2006; 31:465-76. 
[6] Suthisisang C. Tramadol: victims of drug abuse [online]. 2013 [cited 2016 Sept 16]. Available from: http://www.pharmacy.mahidol.ac.th/ th/knowledge/article/161. [in Thai]

[7] Bureau of Information, Office of the Permanent Secretary, Ministry of Public Health, Thailand. Physicians warn the youth that abusing drug can lead to drug addiction and destroy their life [online]. 2016 [cited 2017 May 22]. Available from: https://pr.moph.go.th/?url=pr/ detail/all/02/90409. [in Thai]

[8] Tungtananuwat W, Lawanprasert S. Fatal $4 \times 100$; home-made kratom juice cocktail. J Health Res. 2010; 24:43-7.

[9] Post Today. Caution!! “Taking Pro" lead to hallucination-death [online]. 2015 [cited 2017 May 22]. Available from: $h t t p: / / w w w$. posttoday.com/analysis/report/374392. [in Thai]

[10] Abood EA, Wazaify M. Abuse and misuse of prescription and nonprescription drugs from community pharmacies in Aden City-Yemen. Subst Use Misuse. 2016; 51:942-7.

[11] Ministry of Public Health. Official Document Number 1009.2/12612, signed by secretary general of the Thai Food and Drug Administration, subject: Measures to control the sale of tramadol. 2013 Sept 6. [in Thai]

[12] Thai Visa, The Nation. NCB regulates pharmacies to prevent drug abuse [online]. 2015 [cited 2017 May 23]; Available from: https://www.thaivisa.com/forum/topic/839317-ncb-regulates-thaipharmacies-to-prevent-drug-abuse/

[13] Bureau of Drug Control. Statistics of license related to business of drug trading in Thailand, year 2014 [online]. 2014 [cited 2018 Feb 1]. Available from: http://www.fda.moph.go.th/sites/drug/ download/สถิติ.pdf [in Thai]

[14] Yamane T. Statistics: an introductory analysis. 3rd ed. New York: Harper and Row; 1973.

[15] Department of City Planning, Bangkok Metropolitan Administrator. A land use plan classified to land use category BE 2556, Principle City Planning BE 2556 [online]. 2015 [cited 2018 Feb 1]. Available from: http://cpd.bangkok.go.th:90/web2/NEWCPD2556/02_cpd56. $p d f[$ in Thai]
[16] Pergolizzi JV Jr, van de Laar M, Langford R, Mellinghoff HU, Merchante IM, Nalamachu S, et al. Tramadol/paracetamol fixed-dose combination in the treatment of moderate to severe pain. J Pain Res. 2012; 5:327-46.

[17] Rawal N, Macquaire V, Catalá E, Berti M, Costa R, Wietlisbach M. Tramadol/paracetamol combination tablet for postoperative pain following ambulatory hand surgery: a double-blind, doubledummy, randomized, parallel-group trial. J Pain Res. 2011; 4:103-10.

[18] Raffa RB, Pergolizzi JV Jr, Tallarida RJ. The determination and application of fixed-dose analgesic combinations for treating multimodal pain. J Pain. 2010; 11:701-9.

[19] Sansone RA, Sansone LA. Tramadol: seizures, serotonin syndrome, and coadministered antidepressants. Psychiatry (Edgmont). 2009; 6:17-21.

[20] Lynch T, Price A. The effect of cytochrome P450 metabolism on drug response, interactions, and adverse effects. Am Fam Physician. 2007; 76:391-6.

[21] Zabihi E, Hoseinzaadeh A, Emami M, Mardani M, Mahmoud B, Ali Akbar M. Potential for tramadol abuse by patients visiting pharmacies in northern Iran. Subst Abuse. 2011; 5:11-5.

[22] Bashirian S, Barati M, Fathi Y. Prevalence and factors associated with tramadol abuse among college students in west of Iran: an application of the theory of planned behavior. Avicenna J Neuro Psych Physio. 2014; 1:e20314. doi: 10.17795/ajnpp-20314

[23] Santisetthsin P, Thananithisak C. Public and community pharmacist opinions toward the control measure of tramadol drug dispensing. Srinag Med J. 2014; 29:559-66. [in Thai, English abstract]

[24] Spiller HA, Scaglione JM, Aleguas A, Foster H, Durback-Morris L, Scharman EJ, Baker SD. Effect of scheduling tramadol as a controlled substance on poison center exposures to tramadol. Ann Pharmacother. 2010; 44:1016-21.

[25] United Nations: Office on Drugs and Crime. Schools: school-based education for drug abuse prevention. New York: United Nation Publications; 2004. 